\title{
A method for updating joint parameters in medium-frequency vibrations
}

\author{
Olivier Dorival - Philippe Rouch - Olivier Allix
}

LMT Cachan (ENS Cachan - CNRS - Université Paris 6)

61 avenue du Président Wilson, F-94235 Cachan cedex

\{dorival, rouch, allix\}lmt.ens-cachan.fr

\begin{abstract}
Joints between substructures play a significant role in the vibrational behavior of complex structures because they govern energy flow and most of the dissipative phenomena. In order to identify joint models, this paper proposes a robust updating method which was initially based on studies of the error in constitutive relation in relation to finite element model updating. Here, it is redesigned in order to focus on joint models in medium-frequency problems. In order to do that, we use an alternative numerical approach called the Variational Theory of Complex Rays (VTCR). After introducing the new formulation, the paper analyzes the effectiveness of the approach in identifying a joint's stiffness and damping. RÉSUMÉ. Les liaisons entre sous-structures jouent un rôle crucial dans la réponse vibratoire d'une structure complexe, en régissant les transferts énergétiques ainsi qu'une grande partie de la dissipation. Pour identifier ces modèles de liaison, ce travail s'inspire d'une méthode de recalage robuste, initialement basée sur les travaux sur l'erreur en relation de comportement pour la correction de modèles éléments finis. La formulation est revisitée pour se concentrer sur le problème des jonctions en moyennes fréquences. Dans ce but une approche numérique alternative aux éléments finis, la théorie variationnelle des rayons complexes (TVRC) est utilisée. Après avoir présenté la nouvelle formulation, nous étudions l'efficacité de cette approche pour l'identification des paramètres de raideur et d'amortissement d'une liaison.

KEYWORDS: medium frequency, joints, damping, updating, VTCR, inverse problem, domain decomposition methods.

MOTS-CLÉS : moyennes fréquences, liaisons, amortissement, recalage, TVRC, problème inverse, décomposition de domaine.
\end{abstract}

DOI:10.3166/REMN.17.713-723 @ 2008 Lavoisier, Paris 


\section{Introduction}

The treatment of dissipation is of primary importance in seeking the numerical response of vibrating structures. In many real systems, one can decompose the structure into simple linear viscoelastic substructures and complex, coarsely modeled interfaces. The behavior of joints, which usually involves complex dissipative phenomena (micro/macro frictional contact, microshocks, heating, ...), must be accounted for because in vibration problems energy losses affect the magnitude of the structural response directly. In addition, the stiffness of joints can significantly influence how the injected power is distributed among the different substructures. Regarding the low-frequency range, updating methods for joints have already been the subject of much research (Mottershead et al., 1993; Deraemaeker, 2001); for high frequencies, the identification of coupling loss factors (Maxit et al., 2001) is an unavoidable step of methods derived from Statistical Energy Analysis (SEA) (Lyon, 1975). Between these two extremes, medium frequencies present major difficulties because of their high modal density and their high sensitivity to boundary conditions. This paper presents a method for updating joint parameters - in our case, joint stiffness and damping - with special emphasis on the robustness of this technique.

Different methods are available in order to deal with various types of inverse physical problems (Bonnet et al., 2005). Our method uses the modified error in constitutive relation approach (Ladevèze et al., 1999), which is derived from the well-known concept of error in constitutive relation for the quantification of finite element discretization errors (Ladevèze et al., 1983). Starting with a complete set of available information coming from the numerical model as well as experimental measurements, the method consists in dividing the corresponding equations into two groups according to their reliability. The reliable equations (in relation to both the numerical model and the experimental model) are to be satisfied exactly, while the unreliable equations can be solved approximately through the minimization of a cost function. In the case of a joint modeling problem, the equations of the continuous model are divided as follows: the substructures are considered to be reliable components, while the joint equations - including the model parameters which need to be identified - are unreliable parts of the structure. Regarding the experimental information, the locations and directions of the sensors and the prescribed angular frequency and excitation force are assumed to be reliable. The quantities measured by the sensors, which are unavoidably noisy, are considered unreliable. Table 1 summarizes the characteristics of these different types of information. The solution of the unreliable equations is achieved by minimizing a cost function composed of two terms: a modeling error which quantifies the satisfaction of the joint equations, and an experimental error which represents the discrepancy between the numerical solution and the experimental measurements.

Then, a numerical scheme must be applied in order to solve the problem. In dealing with medium-frequency problems, classical methods encounter serious difficulties. Finite element calculations require the use of a very refined mesh in order to avoid what one calls pollution errors due to the small-wavelength phenomena involved (Ihlenburg et al., 1997); this leads to sharp increases in computational costs. 
High-frequency methods must be modified in order to release some of the SEA assumptions which are no longer valid in the medium-frequency regime. Among the various possible methods (Farhat et al., 2003; Strouboulis et al., 2006), we chose to use the Variational Theory of Complex Rays (VTCR) (Ladevèze et al., 2003), which has been found to be easy to adapt to the separation of the equations discussed previously. This Trefftz method consists in building over each substructure two-scale shape functions which satisfy the dynamic equilibrium and the constitutive law of the substructure exactly. (These equations are considered to be reliable.) The boundary conditions of each subdomain are applied weakly through a variational equation which can be viewed as a balance between virtual powers at the boundaries. In order to distinguish joint equations from other boundary conditions, we use a substructured formulation of the VTCR which was presented in (Dorival et al., 2006). Further details of the formulation and its discretization can be found in (Dorival et al., 2008).

This paper is organized as follows: Section 2 briefly describes the formulation. Section 3 presents the identification of the joint's stiffness and damping parameters for a simple simulated structure, which illustrates the robustness of the method.

Table 1. The proposed method for carrying out joint updating

\begin{tabular}{|c|c|c|}
\hline & Continuous model & Experimental model \\
\hline $\begin{array}{c}\text { Reliable } \\
\text { Information }\end{array}$ & $\begin{array}{l}\text { - Geometry } \\
\text { - Substructure equations: } \\
\text { - local dynamic equilibrium } \\
\text { - constitutive law } \\
\text { - Reliable boundary conditions }\end{array}$ & $\begin{array}{l}\text { - Angular frequency } \\
\text { - Sensor locations } \\
\text { - Sensor directions } \\
\text { - Prescribed forces }\end{array}$ \\
\hline $\begin{array}{c}\text { Unreliable } \\
\text { information }\end{array}$ & $\begin{array}{l}\text { - Joint equations: } \\
\text { - constitutive law of the joint } \\
\text { - dynamic equilibrium of the joint }\end{array}$ & $\begin{array}{c}\text { - Measured amplitudes } \\
\text { (with noise) }\end{array}$ \\
\hline
\end{tabular}

\section{Formulation of the inverse problem using a substructured Trefftz method}

\subsection{A substructured Trefftz method for the equations of the continuous model}

This first section deals with the equations of the continuous model alone. The experimental equations will be introduced in the next section in order to produce an inverse problem. The approach is based on a substructured version of the VTCR which was detailed in (Dorival et al., 2006). For the sake of simplicity, let us consider two substructures $\Omega_{i, i=1,2}$ and denote the displacement field and the stress tensor $\underline{u}_{i}$ and $\underline{\sigma}_{i}$ respectively.

The substructures $\Omega_{i}$ are distinct from the joint $\Gamma$ to be identified (see Figure 1). The joint is considered to be a real substructure with its own equations and interface unknowns denoted $\underline{\hat{W}}_{i}$ and $\underline{\hat{F}}_{i}$ (which are distinct from the unknowns of the adjacent 
substructures). Interface forces $\underline{\hat{F}}_{i}$ are applied to each substructure. The equations of the substructures are considered to be reliable and satisfied exactly. The numerical treatment of each substructure $\Omega_{i}$ involves a classical formulation of the VTCR: the solution within each substructure is sought as a linear combination of complex rays (see Figure 2) which satisfies the local dynamic equilibrium and constitutive law exactly. Boundary conditions are applied in variational form. In practice, the strong physical meaning of the shape functions enables one to use only a very small number of unknowns - about one hundred per substructure. It is important to observe that the numerical cost does not increase with the frequency being considered. Additional details on the VTCR can be found in (Ladevèze et al., 2003). The problems in each substructure $\Omega_{i}$ lead to admissibility constraints associated with admissibility spaces $\mathcal{S}_{a d}^{i}$.

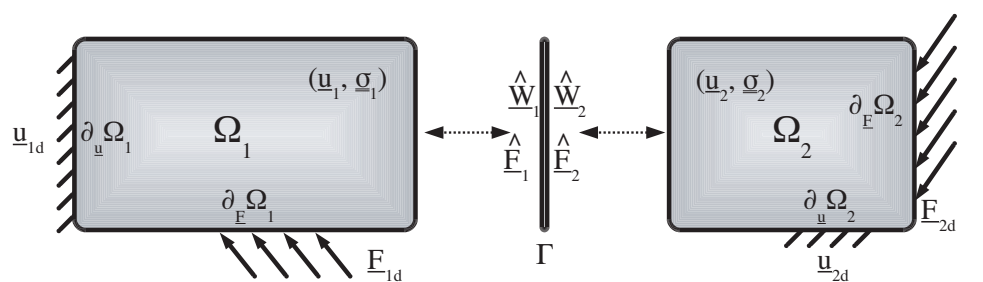

Figure 1. Separation of the joint to be identified from the reliable substructures

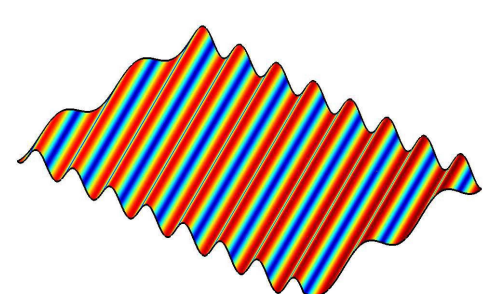

(a)

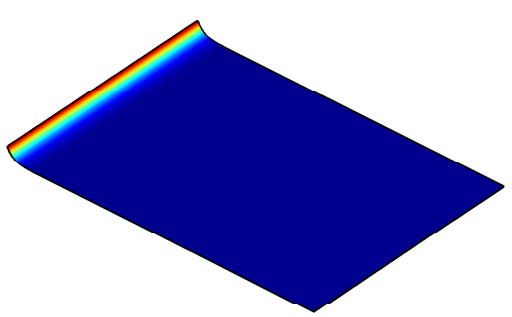

(b)

Figure 2. Examples of complex rays: (a) interior ray and (b) ray localized at an edge

Joint equations with joint unknowns $\underline{\hat{W}}_{i}$ and $\underline{\hat{F}}_{i}$ must be satisfied. In our case, we assume that line spring-mass-dampers are distributed along the interface $\Gamma$. The equations are satisfied by minimizing a residual, called the modeling error, which is constructed as follows:

$$
\begin{aligned}
E_{m d l}^{2}= & \frac{1}{N_{N}} \int_{\Gamma}\left\|\underline{\hat{F}}_{1}+\underline{\hat{F}}_{2}+\frac{m \omega^{2}}{2}\left(\underline{\hat{W}}_{1}+\underline{\hat{W}}_{2}\right)\right\|^{2} d l \\
& +\frac{1}{N_{W}} \int_{\Gamma}\left\|\underline{\hat{W}}_{1}-\underline{\hat{W}}_{2}+\frac{k}{2}\left(\underline{\hat{F}}_{1}-\underline{\hat{F}}_{2}\right)\right\|^{2} d l
\end{aligned}
$$


The line mass $m$ and line stiffness $k$ can vary along the joint and dissipative behavior can be represented as a complex part of $m$ and $k$. Then, the joint unknowns are discretized in the same way as the matching substructure unknowns in order to obtain a conforming discretization.

The continuous problem consists in minimizing the modeling error $E_{m d l}^{2}$ under the admissibility constraints $\mathcal{S}_{a d}^{i}$. This is achieved by solving the corresponding lagrangian problem.

\subsection{Experimental information and the inverse problem}

In order to produce an inverse problem, experiments are performed on the actual structure. Let us designate measured quantities as $\widetilde{\bullet}$. When dealing with mediumfrequency vibrations, the hypersensitivity of the structure requires the use of nonclassical measurement techniques. Indeed, intrusive techniques modify the structure's mass and stiffness locally, and pointwise measurements provide only very local information. Laser vibrometry, which is widely used in high-frequency measurements, is an appealing technique to overcome these difficulties because it is nonintrusive and can provide field measurements capable of capturing the very local phenomena which occur in this frequency range.

Given a set of measurement points $\underline{X}_{m}$, let us define the following measurement error in order to quantify the distance between the simulation and the experimental results:

$$
E_{m e s}^{2}=\frac{1}{N_{m e s}} \sum_{m}\left\|\underline{u}_{\left(\underline{X}_{m}\right)}-\underline{\widetilde{u}}_{\left(\underline{X}_{m}\right)}\right\|^{2}
$$

The summation of the measurement error and the modeling error leads to the modified error:

$$
E_{\text {mod }}^{2}=(1-r) E_{m d l}^{2}+r E_{m e s}^{2}
$$

where $r$ is used to balance the two terms according to one's relative confidence in the model and in the experiments. If $r=0$ is chosen, the modified error only accounts for the interface model. On the contrary if $r=1$, the interface equations have no weight and the solution tries to satisfy the experimental results at best. For $r$ chosen between 0 and 1 , one obtains a solution which is a compromise between the measured data and the joint model chosen by minimizing the modified error under admissibility constraints $\mathcal{S}_{a d}^{i}, i=1,2$. A typical value of 0.8 will be used in Section 3 .

Since the modified error depends on $k$ joint parameters $p_{k}$, the inverse problem consists in finding the optimum joint parameters $p_{k}^{o p t}$ :

$$
\left(p_{k}^{\text {opt }}\right)=\arg \min _{\left(p_{k}\right)} E_{\bmod \left(p_{k}\right)}^{2}
$$

The corresponding nonlinear optimization problem can be solved through classical Newton-like methods. 


\section{Example of the identification of a joint's stiffness and damping}

\subsection{The experimental structure (simulated)}

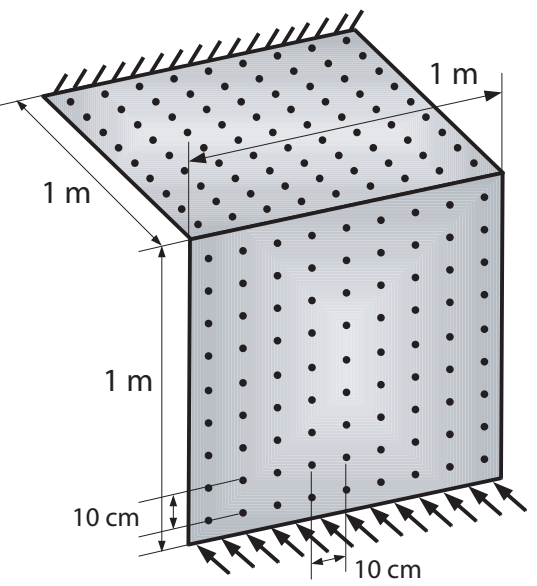

Figure 3. Simulated experimental structure consisting of two plates connected by a joint

Figure 3 presents a very simple structure consisting of two plates connected by a joint. One plate is fixed along an edge and the other is subjected to a harmonic line shear force with angular frequency $\omega=155 \mathrm{~Hz}$. The dots represent measurement points. The experimental data were simulated by a classical VTCR calculation using the following plate properties: Young's modulus $E=210 e 9 \mathrm{MPa}$, Poisson's ratio $\nu=0.3$, plate thickness $h=0.0007 \mathrm{~m}$, mass density $\rho=7,800 \mathrm{~kg} \cdot \mathrm{m}^{-3}$, structural damping $\alpha=0.01$.

For the sake of simplicity, the shear behavior of the joint is assumed to be perfectly rigid and, therefore, the rotational behavior of the joint alone needs to be identified. In our case, the joint's rotational stiffness of the "actual" structure was $k_{0}=10^{1.5} \mathrm{~N} . \mathrm{m}$ and its mass was neglected. Viscous joint damping was assumed with two dissipation levels: a slightly dissipative joint with $\eta=0.1$ and a highly dissipative joint with $\eta=10$. At this frequency, the first case dissipated $9 \%$ of the total energy and the second case $68 \%$. The rotational joint equations are:

$$
\begin{aligned}
\underline{n}_{1} \hat{\mathbf{M}}_{1} \underline{n}_{1}+\underline{n}_{2} \hat{\mathbf{M}}_{2} \underline{n}_{2} & =0 & & \text { moment equilibrium } \\
\underline{n} \overline{\mathbf{M}} \underline{n} & =-k_{0}(1+i \eta) \bar{u}_{, n} & & \text { constitutive law }
\end{aligned}
$$

where $\hat{\mathbf{M}}_{i}$ and $\hat{u}_{i, n}$ respectively denote the bending moment and the normal rotation over $\Gamma$. The overbar notation $\bar{u}_{, n}$ (respectively $\overline{\mathbf{M}}$ ) represents the average value of normal rotations (resp. bending moments) over $\Gamma$. In order to simulate measurement noise, the experimental data were perturbed by $\pm 20 \%$ uniform random noise. 
In the identification process, a classical value of 0.8 was assigned to the weighting parameter $r$. The inverse problem was discretized using 400 complex rays - 64 interior complex rays and $4 \times 9$ edge rays for each plate, and the same for the interface DOFs. In the following section, we investigate the possibility of identifying the joint's stiffness and damping separately. Indeed, a real structure can require the identification of a large number of joints, and the optimization problem [4] can become very costly. Therefore, separate identification can be useful. In addition, for identifiability reasons (Wang et al., 1991), it is advisable to seek the stiffness first, then the damping coefficient. The effectiveness of this approach is studied in the next section.

\subsection{Identification of joint stiffness}

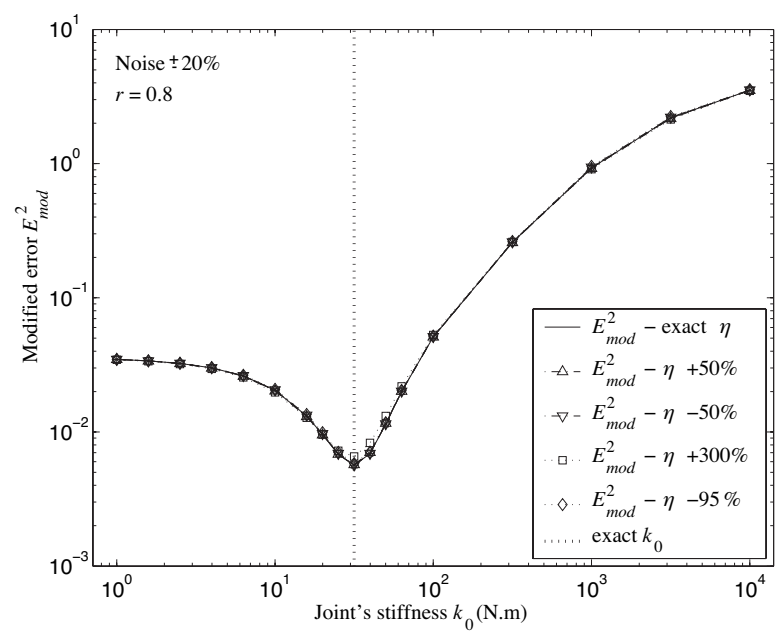

Figure 4. The negligible influence of an erroneous $\eta$ on the minimum of the cost function $E_{\bmod \left(k_{0}\right)}^{2}$ for a slightly dissipative joint. The vertical dotted line represents the exact parameter to be identified (where the minimum should be located)

Figure 4 shows the evolution of the modified error when identifying the stiffness of a slightly dissipative joint $(\eta=0.1)$. The cost function is sufficiently convex to be minimized without difficulty both for exact and highly erroneous damping coefficients. The errors on $\eta$ have no impact: the identified stiffness is very close to the exact stiffness represented by a vertical dotted line. This is easily explained by the fact that for this slightly dissipative joint (in comparison with the substructures' dissipation) the joint's damping coefficient does not play a significant role. Let us note 
that the minimum of the cost function is due to the measurement noise, which has no influence on the location of the minimum because of the design of the cost function and the field measurements.

Figure 5 shows the same results for a highly dissipative joint $(\eta=10)$. With an exact damping coefficient (continuous line), the joint's stiffness is correctly identified. This is not true with an erroneous damping coefficient, in which case the identified stiffness differs from the exact value (vertical dotted line). We conclude that for a highly dissipative joint the damping parameter $\eta$ plays a significant role and must be modeled accurately in order to recover the correct stiffness.

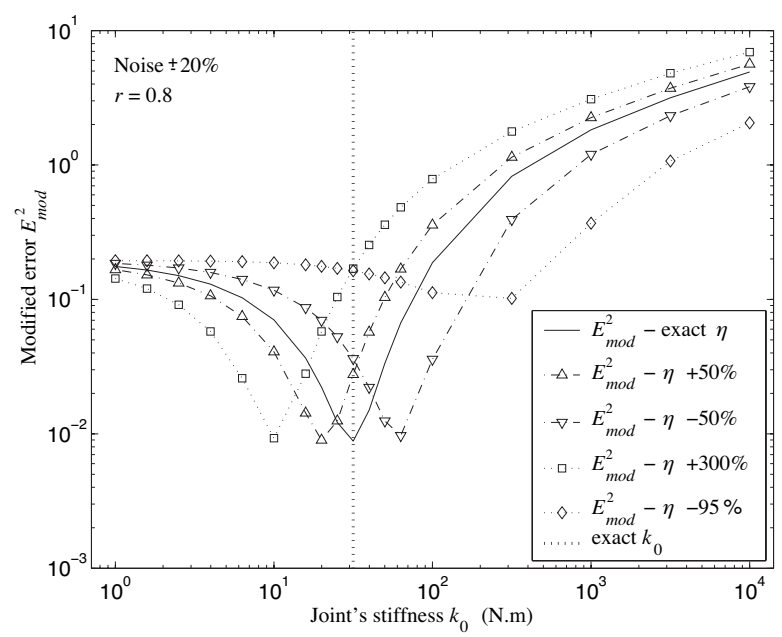

Figure 5. The strong influence of an erroneous $\eta$ on the minimum of the cost function $E_{\text {mod }\left(k_{0}\right)}^{2}$ for a highly dissipative joint. The vertical dotted line represents the exact parameter to be identified (where the minimum should be located)

\subsection{Identification of the joint damping}

In this section, we address the identification of the joint's damping with particular emphasis on the influence of an erroneous joint stiffness. To understand this better, the measurement noise was removed. The evolution of the modified error as a function of the damping parameter $\eta$ in the case the highly dissipative joint is shown in Figure 6. The minimization of the cost function leads to the exact damping parameter, even if the stiffness is highly erroneous. The explanation is quite simple: in the case of a highly dissipative joint (in comparison with the substructures' dissipation), the response is influenced mainly by the damping coefficient, which leads to a very robust identification of $\eta$. 


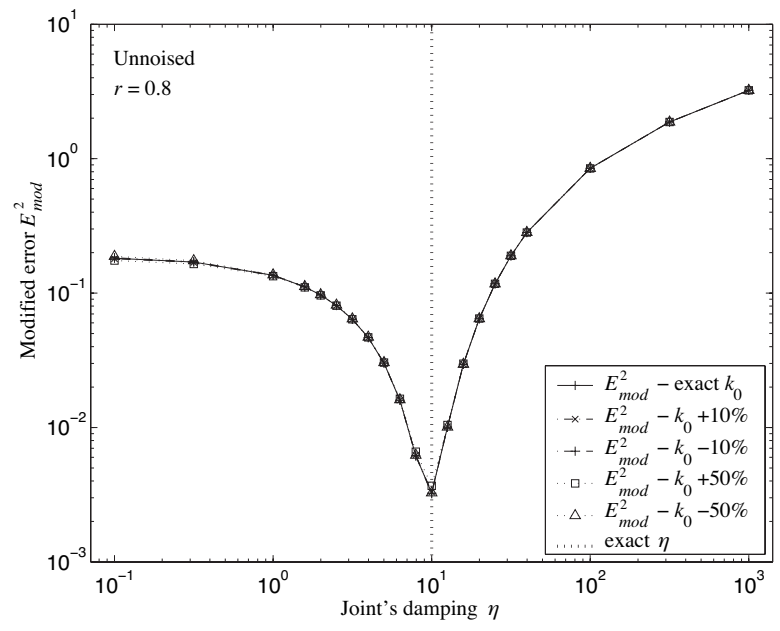

Figure 6. The negligible influence of an erroneous $k_{0}$ on the minimum of the cost function $E_{\text {mod }(\eta)}^{2}$ for a highly dissipative joint. The vertical dotted line represents the exact parameter to be identified (where the minimum should be located)

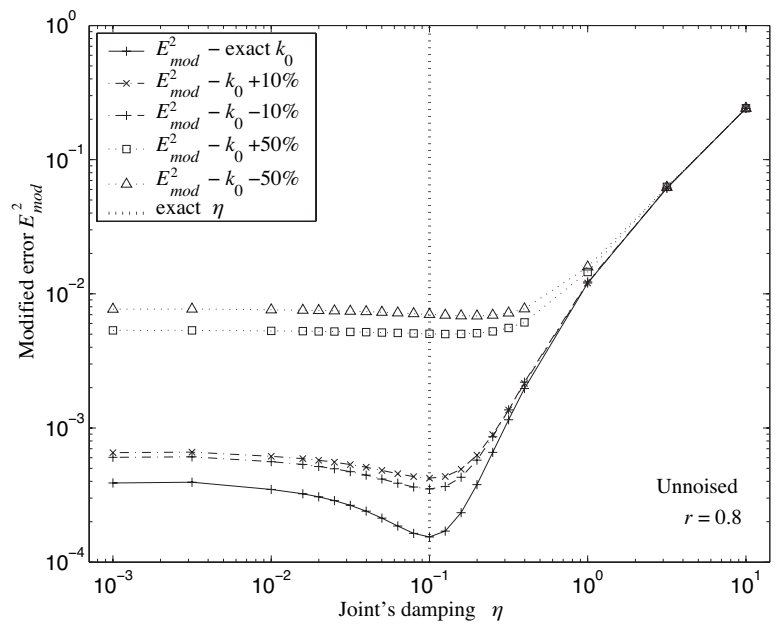

Figure 7. The strong influence of an erroneous $k_{0}$ on the minimum of the cost function $E_{\text {mod (n) }}^{2}$ for a slightly dissipative joint. The vertical dotted line represents the exact parameter to be identified (where the minimum should be located) 
In the case of a slightly dissipative joint (see Figure 7), the evolution of the cost function is less affected by the damping parameter $\eta$. If the stiffness is taken equal to the exact value (continuous line), the damping parameter identified at the minimum of the cost function is exact. Unfortunately, the existence of erroneous joint stiffnesses perturbs this minimum, or even causes it to vanish, which makes the updating process even trickier. In this case, the influence of the damping parameter is not significant enough because the dissipation in the joint is negligible compared to the dissipation in the substructures. Consequently, classical difficulties in the identification of $\eta$ (Wang et al., 1991) seem unavoidable.

\section{Concluding remarks}

In this paper, we presented an updating method for joint models. This technique uses a suitable separation of all information in order to circumvent the difficulties related to medium-frequency vibrations as well as emphasize the behavior of the joints. The numerical approach for discretizing the inverse problem follows exactly the same lines. The robustness of the method was briefly illustrated using a simulated structure. We found that the identification of a joint's stiffness and damping by a step-by-step process cannot be successful if the joint's dissipation is high. In this case, damping should be identified first. This conclusion is a consequence of the sensitivity of the response to the two parameters. At this stage, the experience of the engineer is of great importance for industrial applications. Although the numerical framework developed here turns out to be effective and efficient, further studies should focus on the derivation of a physically consistent experimental part (in the cost function) suitable for medium-frequency phenomena.

\section{References}

Bonnet M., Constantinescu A., “Inverse problems in elasticity”, Inverse Problems, vol. 21, p. R1-R50, 2005.

Deraemaeker A., Sur la maîtrise des modèles en dynamique des structures à partir de résultats d'essais, PhD thesis, Université Paris VI - ENS Cachan, 2001.

Dorival O., Rouch P., Allix O., “ A substructured version of the variational theory of complex rays dedicated to the calculation of assemblies with dissipative joints in the mediumfrequency range", Engineering Computations, vol. 23, n 7, p. 729-748, 2006.

Dorival O., Rouch P., Allix O., “ A substructured Trefftz method for updating joint models in the medium-frequency range”, Computational Mechanics, vol. 42, p. 381-394, 2008.

Farhat C., Harari I., Hetmaniuk U., “ A discontinuous Galerkin method with Lagrange multipliers for the solution of Helmholtz problems in the mid-frequency regime", Comput. Methods Appl. Mech. Engrg., vol. 192, p. 1389-1419, 2003.

Ihlenburg F., Babŭska I., " Finite element solution of the Helmholtz equation with high wave number. Part II: The h-p-version of the FEM", Siam J. of Numer. Anal., vol. 34, n 1, p. 315358, 1997. 
Ladevèze P., Chouaki A., “ Application of a posteriori error estimation for structural model updating”, Inverse Problems, vol. 15, p. 49-58, 1999.

Ladevèze P., Leguillon D., “ Error estimate procedure in the finite element method and application”, SIAM J. Numer. Anal., vol. 20, n 3, p. 485-509, 1983.

Ladevèze P., Rouch P., Riou H., Bohineust X., “ Analysis of medium-frequency vibrations in a frequency range", Jnl of Comp. Acoustic, vol. 11, n 2, p. 255-283, 2003.

Lyon R. H., Statistical Energy Analysis of dynamical systems: theory and applications, MIT Press, Cambridge, 1975.

Maxit L., Guyader J.-L., " Estimation of SEA coupling loss factors using a dual formulation and FEM modal information, Part I: theory", Journal of Sound and Vibration, vol. 239, n 5 , p. 907-930, 2001.

Mottershead J., Friswell M., “ Model updating in structural dynamics: a survey”, Journal of Sound and Vibration, vol. 167, n 2 2, p. 347-375, 1993.

Strouboulis T., Babuska I., Hidajat R., “ The Generalized Finite Element Method for Helmholtz equation: theory, computations and open problems", Comput. Meth. Appl. Mech. Engrg., vol. 195, p. 4711-4731, 2006.

Wang J., Liou C., “ Experimental identification of mechanical joint parameters”, Journal of Vibration and Acoustics, vol. 113, p. 28-36, 1991. Transaction of the ASME. 
